\title{
Variants in HNRNPDL and SETX Not Necessarily Indicate Familial Amyotrophic Lateral Sclerosis or Limb Girdle Muscular Dystrophy 1G in Acute Muscular Respiratory Failure
}

\author{
Josef Finsterer ${ }^{1}$ Claudia Stöllberger ${ }^{2}$ Hans Keller $^{2}$ \\ ${ }^{1}$ Krankenanstalt Rudolfstiftung, Messerli Institute, Vienna, Austria \\ 2nd Medical Department with Cardiology and Intensive Care \\ Medicine, Krankenanstalt Rudolfstiftung, Vienna, Austria \\ ${ }^{3}$ Institute of Medical Genetics, Medical University of Vienna, \\ Vienna, Austria
}

J Neurosci Rural Pract 2020;11:353-354

Address for correspondence Josef Finsterer, MD, PhD, Postfach 20, Vienna, 1180, Austria (e-mail: fifigs1@yahoo.de).

\begin{abstract}
Genetic work-up is useful for the identification of a primary myopathy. However, even sophisticated genetic methods may fail to detect the underlying cause of myopathy as in the following case. The patient is a 52-year-old female with a history of epilepsy, arterial hypertension, atrial flutter requiring cardioversion, ablation, and anticoagulation, coronary heart disease, hyperlipidemia, and hyper-CKemia. At age 52 years,

Keywords

- amyotrophic lateral sclerosis

- genetics

- mitochondria

- mitochondrial disorders

- neuromuscular disorders she was referred for heart failure due to ischemic cardiomyopathy requiring appropriate medication and implantation of an ICD. During hospitalization she developed acute muscular respiratory failure requiring mechanical ventilation. Genetic panels for myopathy, neuropathy, and cardiomyopathy revealed variants of unknown significance in the HNRNPDL and SETX genes respectively. Clinical presentation and muscle biopsy, however, suggested metabolic myopathy. Acute muscular respiratory failure may require traditional diagnostic work-up for primary myopathy and long-term invasive and non-invasive ventilation. Panel investigations not necessarily lead to a conclusive diagnosis. The multisystem nature of the condition rather suggests a metabolic defect than LGMD-1G or fALS as genetic findings suggested.
\end{abstract}

\section{Case Report}

The patient is a 52-year-old female, height $160 \mathrm{~cm}$, who was referred for heart failure. Her history included recurrent syncopes/seizures in childhood, pyelonephritis in adolescence, diabetes, arterial hypertension, hyperCKemia, coronary heart disease, atrial flutter requiring cardioversion, ablation, and anticoagulation, non-ST segment myocardial infarction, hyperlipidemia, statin myopathy, and smoking 35 pack/years. The family history was positive for dementia and statin myopathy (mother).

Upon bisoprolol, eplerenone, sacubitril/valsartan, and furosemide, heart failure resolved. Creatine-kinase did not exceed 899U/L $(n<170 U / L)$. Electrocardiogram showed left-anterior hemiblock and right bundle-branch block. Echocardiography revealed systolic and diastolic dysfunction, ventricular hypertrophy, biatrial enlargement, right ventricular dilatation, and noncompaction. Cardiac magnetic resonance imaging confirmed echocardiographic findings and additionally revealed transmural late gadolinium-enhancement. For primary prophylaxis, an implantable cardioverter-defibrillator (ICD) was implanted. ICD-implantation was complicated by a toxic reaction to local anesthetics. Nonalcoholic liver cirrhosis was detected.

On hospital day (hd) 17, she developed acute, muscular respiratory failure under continuous positive airway pressure-assisted spontaneous breathing (CPAP-ASB) requiring 
intubation/mechanical ventilation. Clinical neurologic exam revealed hypermetropia, weakness for head anteflexion (M5-), weakness for elbow extension (M5-), reduced tendon reflexes, and male hair-type. Nerve-conduction studies revealed axonal neuropathy. Muscle biopsy revealed selective type-2 fiber atrophy, occasional COX-negative fibers, and deposition of complement complexes. Genetic panel for myopathy, neuropathy, and cardiomyopathy revealed the variants c. $115 \mathrm{G}>\mathrm{C}$ and c.1327G $>\mathrm{T}$ of unknown significance in HNRNPDL and SETX, respectively.

Extubation was unsuccessful two times and followed by a third intubation on hd 33 and tracheotomy. On hd 110, the patient was released with noninvasive ventilation (NIV) by a home-respirator. After a period of altogether 10 months, she could be weaned off NIV.

Despite a panel for myopathy, neuropathy, and cardiomyopathy, the cause of cardiomyopathy and primary myopathy could not be identified. HNRNPDL variants have been associated with autosomal-dominant limb girdle muscular dystrophy- $1 \mathrm{G}$ and SETX variants have been associated with familial amyotrophic lateral sclerosis. However, the clinical presentation was neither compatible with limb girdle muscular dystrophy-1G $\mathrm{G}^{1}$ nor with familial amyotrophic lateral sclerosis. ${ }^{2}$ Arguments in favor of a metabolic myopathy are previous reports about acute respiratory failure as a complication of metabolic myopathy, ${ }^{3,4}$ the multisystem disease, the mother's history positive for statin-induced myopathy and dementia, deterioration of muscle weakness upon local anesthestics, statin-intolerance, noncompaction, and incompatibility of the variants with the patient's phenotype. Recovery from muscular respiratory failure may be attributed to ventilatory treatment and care, ${ }^{5}$ heart failure therapy, exemplary compliance, or the reversible nature of muscle weakness.

This case shows that acute muscular respiratory failure may require traditional diagnostic work-up and long-term invasive/noninvasive ventilation, and that panel investigations not necessarily lead to a conclusive diagnosis.

\section{Authors' Contributions}

All authors contributed equally. J.F. and C.S. helped in clinical investigations, design, literature search, discussion, first draft, and critical comments.

\section{Funding}

None.

\section{Conflict of Interest}

None declared.

\section{References}

1 Berardo A, Lornage X, Johari M, et al. HNRNPDL-related muscular dystrophy: expanding the clinical, morphological and MRI phenotypes. J Neurol 2019;266(10):2524-2534

2 Brenner D, Weishaupt JH. Update on amyotrophic lateral sclerosis genetics. Curr Opin Neurol 2019;32(5):735-739

3 Koo P, Sethi JM. Metabolic myopathies and the respiratory system. Clin Chest Med 2018;39(2):401-410

4 Boentert $\mathrm{M}$, Prigent $\mathrm{H}$, Várdi $\mathrm{K}$, et al. Practical recommendations for diagnosis and management of respiratory muscle weakness in late-onset pompe disease. Int J Mol Sci 2016;17(10):E1735

5 Benditt JO. Respiratory care of patients with neuromuscular disease. Respir Care 2019;64(6):679-688 